International Journal of Modern Physics A

(C) World Scientific Publishing Company

\title{
ANALYTIC INVARIANT CHARGE AND THE LATTICE STATIC QUARK-ANTIQUARK POTENTIAL
}

\author{
A.V. NESTERENKO \\ Centre de Physique Théorique de l'École Polytechnique \\ 91128 Palaiseau Cedex, France* \\ nesterav@cpht.polytechnique.fr \\ Bogoliubov Laboratory of Theoretical Physics, Joint Institute for Nuclear Research \\ Dubna 141980, Russian Federation \\ nesterav@thsun1.jinr.ru
}

Received 12 December 2003

\begin{abstract}
A recently developed model for the QCD analytic invariant charge is compared with quenched lattice simulation data on the static quark-antiquark potential. By employing this strong running coupling one is able to obtain the confining quark-antiquark potential in the framework of the one-gluon exchange model. To achieve this objective a technique for evaluating the integrals of a required form is developed. Special attention is paid here to removing the divergences encountered the calculations. All this enables one to examine the asymptotic behavior of the potential at both small and large distances with high accuracy. An explicit expression for the quark-antiquark potential, which interpolates between these asymptotics, and satisfies the concavity condition, is proposed. The derived potential coincides with the perturbative results at small distances, and it is in a good agreement with the lattice data in the nonperturbative physically-relevant region. An estimation of the parameter $\Lambda_{\mathrm{QCD}}$ is obtained for the case of pure gluodynamics. It is found to be consistent with all the previous estimations of $\Lambda_{\mathrm{QCD}}$ in the framework of approach in hand.
\end{abstract}

Keywords: Nonperturbative QCD; analytic approach; quark confinement.

\section{Introduction}

The description of hadron dynamics at large distances remains a crucial challenge of elementary particle physics for a long time. On the one hand, the asymptotic freedom of Quantum Chromodynamics (QCD) allows one to apply perturbation theory to study some "short-range" phenomena, for instance, the lowest-lying bound states of heavy quark-antiquark systems. On the other hand, for the consistent description of many phenomena related to the "long-range" dynamics (such as confinement of quarks, structure of the QCD vacuum, etc.) something more than the usual perturbative approach has to be involved.

*Unité Mixte de Recherche du CNRS (UMR 7644) 
Theoretical analysis of the strong interaction basically relies upon the renormalization group (RG) method. Usually, in order to describe the hadron dynamics in the asymptotical ultraviolet (UV) region, one applies the RG method together with perturbative calculations. However, this leads to unphysical singularities of the outcoming solutions to RG equations, that contradicts the general principles of local Quantum Field Theory (QFT). An effective way to overcome such difficulties consists in invoking the analyticity requirement. This prescription became the underlying idea of the so-called analytic approach to QFT, ${ }^{12}$ which was lately extended to QCD by Shirkov and Solovtsov 34

In the framework of the analytic approach to QCD a new model for the strong running coupling has recently been developed!56 Its basic idea is to impose the analyticity requirement on the RG $\beta$ function perturbative expansion for restoring its correct analytic properties (see Sec. 2 for the details). The analytic invariant charge possesses a number of profitable properties, in particular, it contains no unphysical singularities (see also Refs. 7, 8, 9, for the description of its traits). It is worth noting here that this analytic running coupling incorporates the perturbative and intrinsically nonperturbative features of the strong interaction, and enables one to describe a wide range of hadron processes.

A crucial insight into the nonperturbative aspects of the strong interaction at large distances can be provided by lattice simulations. This may be, for instance, the static quark-antiquark $(\mathrm{Q} \overline{\mathrm{Q}})$ potential calculated up to the significantly large distances 10$](r \gtrsim 1 \mathrm{fm})$, or the investigation of the topological structure of the QCD vacuum. 11 Certainly, a decisive test of any model for the strong interaction is its comparison with the lattice results.

The objective of this paper is to study the asymptotic behavior of the static quark-antiquark potential constructed by making use of the analytic invariant charge. It is also of a primary interest to collate the derived $\mathrm{Q} \overline{\mathrm{Q}}$ potential with the relevant lattice simulation data. For verification the consistency of the results it is worth to evaluate the corresponding parameter $\Lambda_{\mathrm{QCD}}$ and to compare it with its previous estimations obtained in the framework of approach in hand.

The layout of the paper is as follows. In Sec. 2 the model for the QCD analytic invariant charge is briefly described and its basic features are outlined. In Sec. 3 the asymptotic behavior of the quark-antiquark potential, constructed by making use of this strong running coupling, is examined. A technique for treating the divergent integrals encountered is developed here. The explicit expression for the $\mathrm{Q} \overline{\mathrm{Q}}$ potential, which possesses the obtained asymptotics, and satisfies the concavity condition, is derived. In Sec. 4 these results are applied to study of the relevant lattice simulation data. The constructed quark-antiquark potential agrees fairly well with the lattice data in the nonperturbative physically-relevant region. At the same time, it coincides with the perturbative $\mathrm{Q} \overline{\mathrm{Q}}$ potential at small distances. The fitted parameter $\Lambda_{\mathrm{QCD}}$ is found to be in a good agreement with all its previous estimations, implying the consistency of obtained results. In the Conclusion (Sec. 5) the achieved goals are formulated in a compact way. 


\section{The QCD Analytic Invariant Charge}

As it has been mentioned in the Introduction, the perturbative approximation of the $\beta$ function in the RG equation for the strong running coupling $\alpha\left(\mu^{2}\right)=g^{2}\left(\mu^{2}\right) /(4 \pi)$

$$
\frac{d \ln \left[g^{2}\left(\mu^{2}\right)\right]}{d \ln \mu^{2}}=\beta\left(g\left(\mu^{2}\right)\right)
$$

leads to unphysical singularities of the outcoming solutions, that contradicts the general principles of local Quantum Filed Theory. A plausible way to overcome such difficulties consists in imposing the analyticity requirement on the perturbative expansion of the RG $\beta$ function for restoring its correct analytic properties. This prescription is a distinctive feature of the recently developed model for the QCD an-

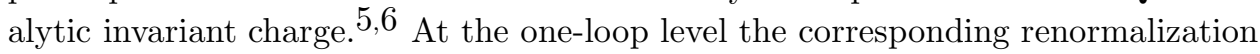
group equation can be solved explicitly:

$$
\alpha_{\mathrm{an}}\left(q^{2}\right)=\frac{4 \pi}{\beta_{0}} \frac{z-1}{z \ln z}, \quad z=\frac{q^{2}}{\Lambda^{2}},
$$

while at the higher loop levels only the integral representation for the analytic invariant charge has been obtained (see Refs. 6 9). Figure 1 presents the analytic running coupling $\widetilde{\alpha}_{\text {an }}\left(q^{2}\right)=\alpha_{\text {an }}\left(q^{2}\right) \beta_{0} /(4 \pi)$ at different loop levels.

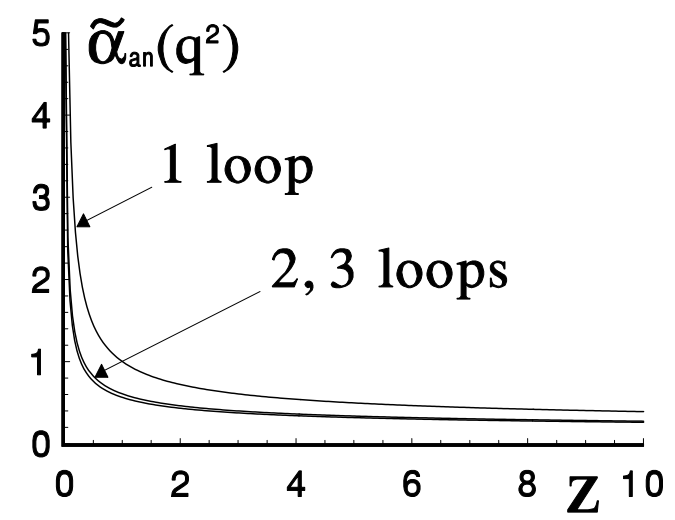

Fig. 1. The QCD analytic invariant charge at different loop levels, $z=q^{2} / \Lambda^{2}$.

The developed model for the QCD analytic invariant charge possesses a number of appealing traits (see Refs. 8, 9). Namely, it has no unphysical singularities at any loop level; it contains no adjustable parameters; it incorporates ultraviolet asymptotic freedom with infrared (IR) enhancement in a single expression; it has universal behavior both in UV and IR regions at any loop level; it possesses a good higher loop and scheme stability. This model also enables one to describe various strong interaction processes both of perturbative and intrinsically nonperturbative nature (see papers 17, 12, 13 and references therein). It is of a primary importance to mention here that the analytic invariant charge (2) has recently been rediscovered 
when studying the conformal inversion symmetry related to the size distribution of instantons. 14 In particular, Eq. (2) was proved to reproduce explicitly this kind of symmetry. In turn, the latter is in a good agreement with the relevant lattice data by the UKQCD Collaboration 11 (see Refs. 14 and 7 for the details).

\section{The Static Quark-Antiquark Potential}

Let us proceed now to the construction of the static quark-antiquark potential. In the framework of the one-gluon exchange model it is related to the strong running coupling $\alpha\left(q^{2}\right)$ by the 3 -dimensional Fourier transformation

$$
V(r)=-\frac{16 \pi}{3} \int_{0}^{\infty} \frac{\alpha\left(\boldsymbol{q}^{2}\right)}{\boldsymbol{q}^{2}} \frac{\exp (i \boldsymbol{q} \boldsymbol{r})}{(2 \pi)^{3}} d \boldsymbol{q} .
$$

Strictly speaking, this definition of the potential is justified for small distances $(r \lesssim 0.1 \mathrm{fm})$ only. For example, the lowest-lying bound states of heavy quarks can be described by employing the perturbative ${ }^{\text {Q }}$ QCD 15 However, at large distances $(r \gtrsim 0.5 \mathrm{fm})$, which play the crucial role in hadron spectroscopy, the perturbative approach blows up due to unphysical singularities (such as the Landau pole) of the strong running coupling. Nevertheless, the model (3), being complemented with a certain insight into the nonperturbative behavior of the QCD invariant charge, has proved to be successful for description both heavy-quark and light-quark systems (see, e.g., reviews 16, 17, 18 and references therein for the details).

In this paper, for the construction of the static potential of the quark-antiquark interaction, we shall use the analytic invariant charge (2). After integration over angular variables, Eq. (3) in this case takes the form

$$
V(r)=-\frac{32}{3 \beta_{0}} \frac{1}{r_{0}} \int_{0}^{\infty} \frac{p^{2}-1}{p^{2} \ln p^{2}} \frac{\sin (p R)}{p R} d p,
$$

where $p=q r_{0}, R=r / r_{0}$, and $r_{0}$ is a reference scale of the dimension of length, which will be specified below. This integral diverges at the lower limit, that is a common feature of the models of such kind (see, e.g., Ref. 16). We shall treat this divergence by employing the analytical regularization (see Eq. (10)).

The analysis of Eq. (4), performed in Ref. [5] elucidated only the leading asymptotic behavior of $V(r)$ at large distances. However, it is still desirable to examine at a more precise level the asymptotics of the quark-antiquark potential (4) in the both, ultraviolet and infrared regions. This objective can be achieved in the following way. First of all, let us introduce a dimensionless variable $Q=p R$ and rewrite

\footnotetext{
aThe leading short-distance nonperturbative effect due to the gluon condensate has also been taken into account in Ref. 15
} 
Eq. (4):

$$
V(r)=\frac{16}{3 \beta_{0}} \frac{1}{r_{0}} \frac{1}{R} \int_{0}^{\infty}\left(1-\frac{R^{2}}{Q^{2}}\right) \frac{\sin Q}{Q} \frac{d Q}{\ln R-\ln Q} .
$$

Then, formally expanding the denominator of the integrand, ${ }^{\mathrm{b}}$ one can find the asymptotic behavior of $V(r)$ at both small and large distances:

$$
\begin{aligned}
V(r) \simeq & \frac{16}{3 \beta_{0}} \frac{1}{r_{0}}\left[\frac{1}{R} \sum_{n=0}^{n_{0}} \frac{1}{(\ln R)^{n+1}} \int_{0}^{\infty} \frac{\sin Q}{Q}(\ln Q)^{n} d Q\right. \\
& \left.-R \sum_{m=0}^{m_{0}} \frac{1}{(\ln R)^{m+1}} \int_{0}^{\infty} \frac{\sin Q}{Q^{3}}(\ln Q)^{m} d Q\right] .
\end{aligned}
$$

The values of $n_{0}$ and $m_{0}$ will be specified below.

For evaluation of the expansion coefficients in Eq. (6) it is worth considering an auxiliary integral of the form:

$$
\int_{0}^{\infty} Q^{t} \sin Q d Q=\sqrt{\pi} 2^{t} \frac{\Gamma\left(1+\frac{t}{2}\right)}{\Gamma\left(\frac{1}{2}-\frac{t}{2}\right)} .
$$

Differentiating this equation $n$ times with respect to variable $t$ we obtain

$$
\int_{0}^{\infty} Q^{t}(\ln Q)^{n} \sin Q d Q=v(n, t)
$$

where

$$
v(n, t)=\sqrt{\pi} \frac{d^{n}}{d t^{n}}\left[2^{t} \frac{\Gamma\left(1+\frac{t}{2}\right)}{\Gamma\left(\frac{1}{2}-\frac{t}{2}\right)}\right] .
$$

In Eq. (7]) the parameter $t$ takes the values $0 \leq|\operatorname{Re}(t+1)|<1$ (see, e.g., Ref. [20). However, in order to determine the expansion coefficients of the second sum in Eq. (6), one has to go to the point $t=-3$, which is outside of this range. Nevertheless, it can be done by making use of the analytic continuation of the left-hand side of Eq. (7). This continuation is unique and it is defined obviously by the right-hand side of Eq. (7) all over the complex $t$-plane except for the points $t=-2 N$, with $N$ being a natural number. Fortunately, we are not dealing with these values of the parameter $t$, and we can put

$$
\int_{0}^{\infty} \frac{\sin Q}{Q^{s}}(\ln Q)^{n} d Q=\left.v(n, t)\right|_{t=-s}, \quad s \neq 2 N \quad(N=1,2,3, \ldots) .
$$

${ }^{\mathrm{b}} \mathrm{A}$ similar method has also been used in Ref. 19 
It should be noted here that this analytical continuation plays the role of regularization of the expansion coefficients in Eq. (6). It is also convenient to introduce the notations

$$
u_{n}=\left.\frac{2}{\pi} v(n, t)\right|_{t=-1}, \quad \omega_{m}=-\left.\frac{2}{\pi} v(m, t)\right|_{t=-3} .
$$

A few first coefficients (11) have a quite simple form, namely $u_{0}=1, u_{1}=-\gamma$, $u_{2}=\gamma^{2}+\pi^{2} / 12$; and $\omega_{0}=1 / 2, \omega_{1}=3 / 4-\gamma / 2, \omega_{2}=\gamma^{2} / 2-3 \gamma / 2+\pi^{2} / 24+7 / 4$, where $\gamma \simeq 0.57721 \ldots$ denotes the Euler's constant (see Ref. 7 ). It is worth noting here that the leading coefficients $u_{0}, \omega_{0}$, and $\omega_{1}$ have also been calculated in Ref. 5 . but by making use of another technique.

Thus, the static quark-antiquark potential (6) can be represented now in the following form:

$$
V(r) \simeq \frac{8 \pi}{3 \beta_{0}} \frac{1}{r_{0}}\left[\frac{1}{R} \sum_{n=0}^{n_{0}} \frac{u_{n}}{(\ln R)^{n+1}}+R \sum_{m=0}^{m_{0}} \frac{\omega_{m}}{(\ln R)^{m+1}}\right], \quad R=\frac{r}{r_{0}} .
$$

At small distances the potential (12) possesses the standard behavior, determined by the asymptotic freedom

$$
V(r)=\frac{8 \pi}{3 \beta_{0}} \frac{1}{r_{0}} \frac{1}{R \ln R}, \quad r \rightarrow 0 .
$$

At the same time, it proves to be rising at large distances

$$
V(r)=\frac{8 \pi}{3 \beta_{0}} \frac{1}{r_{0}} \frac{R}{2 \ln R}, \quad r \rightarrow \infty,
$$

implying the confinement of quarks. It is of a particular interest to mention here that a similar rising behavior of the $\mathrm{Q} \overline{\mathrm{Q}}$ potential has been proposed 21 a long time ago proceeding from the phenomenological assumptions.

Equation (12) describes the behavior of the static quark-antiquark potential (4) at small and large distances. However, its straightforward extrapolation to all distances encounters poles of different orders at the point $R=1$, which apparently is an artifact of the expansion (6). For the practical purposes it would be undoubtedly useful to derive an explicit expression for the $\mathrm{Q} \overline{\mathrm{Q}}$ potential applicable for $0<r<\infty$.

In order to construct an explicit interpolating formula for the quark-antiquark potential (4) we shall employ here the following method. Let us modify the expansion (12) in a "minimal" way, by adding terms which only subtract the singularities at the point $R=1$ and do not contribute to the derived asymptotics. This leads to the following expression for the static quark-antiquark potential

$$
V(r)=\frac{8 \pi}{3 \beta_{0}} \frac{1}{r_{0}}\left\{\sum_{n=0}^{n_{0}} u_{n}\left[\frac{1}{R(\ln R)^{n+1}}\right]_{\mathrm{Reg}}+\sum_{m=0}^{m_{0}} \omega_{m}\left[\frac{R}{(\ln R)^{m+1}}\right]_{\mathrm{Reg}}\right\},
$$

where $R=r / r_{0}$, and at the leading orders $[1 /(R \ln R)]_{\operatorname{Reg}}=1 /(R \ln R)-1 / P$, $\left[1 /\left(R(\ln R)^{2}\right)\right]_{\operatorname{Reg}}=1 /\left(R(\ln R)^{2}\right)-1 / P^{2}$, and $[R / \ln R]_{\operatorname{Reg}}=R / \ln R-1 / P$, $\left[R /(\ln R)^{2}\right]_{\operatorname{Reg}}=R /(\ln R)^{2}-2 / P-1 / P^{2}, P=R-1$ (see also Ref. 7 ). 


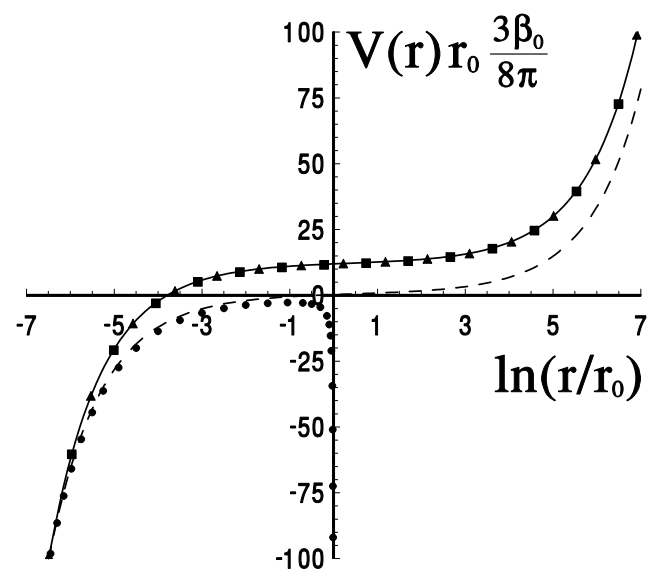

Fig. 2. The quark-antiquark potential 15 in dimensionless units at different levels of approximation: $n_{0}=m_{0}=0$ (dashed curve), $n_{0}=m_{0}=4$ (solid curve), $n_{0}=m_{0}=5(\boldsymbol{\Lambda})$, and $n_{0}=m_{0}=10(\mathbb{\square})$. The one-loop perturbative result is shown by dotted curve.

The numerical analysis of Eq. (15) revealed that for practical purposes it is enough to retain the first five expansion terms $\left(n_{0}=m_{0}=4\right)$ therein. It turns out that the potential (15) itself and the corresponding estimation of the value of parameter $\Lambda_{\mathrm{QCD}}$ are not affected by higher-order contributions. In particular, the curves (15) for $n_{0}=m_{0}=5$ and for $n_{0}=m_{0}=10$ are practically indistinguishable of the curve corresponding to $n_{0}=m_{0}=4$ over the whole region $0<r<\infty$ (see Figure (2). And the higher-order estimations of the parameter $\Lambda_{\mathrm{QCD}}$ vary within $0.5 \%$ of the value obtained at the $n_{0}=m_{0}=4$ (see Sec. 4 further).

Thus, we arrive at the following explicit expression for the static quark-antiquark potential:

$$
\begin{aligned}
V(r)= & V_{0}+\frac{8 \pi}{3 \beta_{0}} \frac{1}{r_{0}}\left\{\frac{1}{R}\left[\frac{1}{\ln R}-\frac{0.577}{(\ln R)^{2}}+\frac{1.156}{(\ln R)^{3}}-\frac{4.021}{(\ln R)^{4}}+\frac{15.018}{(\ln R)^{5}}\right]\right. \\
& +R\left[\frac{0.500}{\ln R}+\frac{0.461}{(\ln R)^{2}}+\frac{1.462}{(\ln R)^{3}}+\frac{3.185}{(\ln R)^{4}}+\frac{17.844}{(\ln R)^{5}}\right] \\
& \left.+\frac{21.489}{1-R}-\frac{62.484}{(1-R)^{2}}+\frac{98.694}{(1-R)^{3}}-\frac{84.143}{(1-R)^{4}}+\frac{32.861}{(1-R)^{5}}\right\},
\end{aligned}
$$

where $R=r / r_{0}$. In order to reproduce the correct short-distance behavior of the $\mathrm{Q} \overline{\mathrm{Q}}$ potential, the dimensional parameter $r_{0}$ in this equation has to be identified with $\Lambda_{\overline{\mathrm{MS}}}$ by the relation $r_{0}^{-1}=\Lambda \exp (\gamma)$ (see, e.g., Refs. 22, 23, 24). It is straightforward to verify that the potential (16) satisfies also the concavity condition

$$
\frac{d V(r)}{d r}>0, \quad \frac{d^{2} V(r)}{d r^{2}} \leq 0
$$

which is a general property of the gauge theories (see Ref. 25] for the details). 


\section{Discussion}

In this section we are going to apply the obtained results to the study of recent quenched lattice simulation data $\frac{10}{10}$ on the static quark-antiquark potential. In particular, the value of the parameter $\Lambda_{\mathrm{QCD}}$ will be estimated by comparing the derived expression for the $\mathrm{Q} \overline{\mathrm{Q}}$ potential (16) with these lattice data.

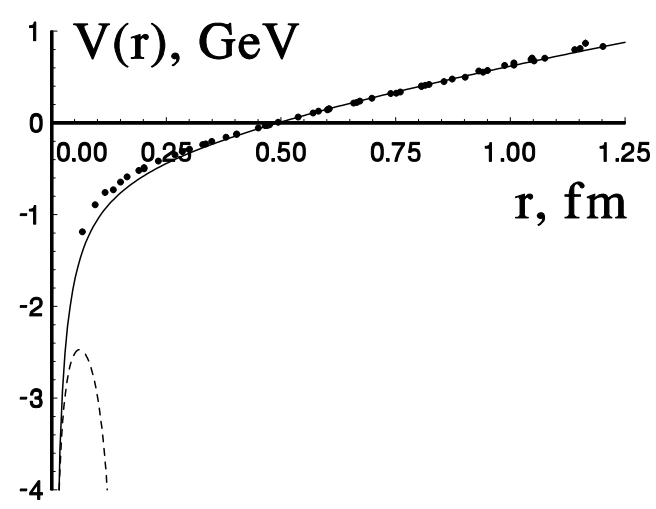

Fig. 3. Comparison of the quark-antiquark potential $V(r)$ defined by Eq. (16) (solid curve) with the quenched lattice simulation datd $10(\bullet)$. The values of the parameters are: $\Lambda=670 \mathrm{MeV}, n_{\mathrm{f}}=0$, $V_{0}=-3.164 \mathrm{GeV}$. The dashed curve corresponds to the relevant one-loop perturbative result.

Thus, a fit of the quark-antiquark potential (16) to the quenched lattice simulation data $\sqrt{10}$ has been performed with the use of the least square method. The varied parameters in Eq. (16) were $r_{0}$ and the additive self-energy constant $V_{0}$. The result of the fit is presented in Figure 3 This figure shows that in the nonperturbative physically-relevant range $0.3 \mathrm{fm} \lesssim r \lesssim 1.2 \mathrm{fm}$, in which the average quark separations $\sqrt{\left\langle r^{2}\right\rangle}$ for quarkonia sits, $\frac{26]}{\text { the }} \mathrm{Q} \overline{\mathrm{Q}}$ potential (16) reproduces the lattice data 10 fairly well. At the same time, in the region $r \lesssim 0.05 \mathrm{fm}$ the derived potential coincides with the perturbative result ${ }^{c}$ (dashed curve in Fig. 3). The difference between the lattice data and the expression (16) in the intermediate range $0.05 \mathrm{fm} \lesssim r \lesssim 0.3 \mathrm{fm}$ may be explained by the presence of additional nonperturbative contributions at these distances (see, e.g., Refs. 29, 30). But the detailed investigation of this matter is beyond the scope of the paper.

Thus, the estimation of the parameter $\Lambda_{\mathrm{QCD}}$ in the course of this comparison gives $\Lambda^{\left(n_{\mathrm{f}}=0\right)}=(670 \pm 8) \mathrm{MeV}$ (this value corresponds to the one-loop level with $n_{\mathrm{f}}=0$ active quarks). The uncertainty here has been calculated by making use of the " $3 \sigma$-criterion" (see Ref. 31). It is worth noting that the fit with the use of the maximum likelihood method results in even better reproduction of the lattice data and gives a similar value $\Lambda^{\left(n_{\mathrm{f}}=0\right)} \simeq 645 \mathrm{MeV}$. Further, in order to collate the

${ }^{\mathrm{c}}$ The reliability of the perturbative quark-antiquark potential at small distances has been discussed in Refs. 23] 27, 28 
obtained estimation with the earlier ones, one has to continue it to the region of three active quarks. It was performed by employing the matching procedure, and gives $^{\mathrm{d}}$ the value $\Lambda^{\left(n_{\mathrm{f}}=3\right)}=(590 \pm 10) \mathrm{MeV}$. The latter is in a good agreement with all the previous estimations of this parameter $\left.\frac{613}{\left[\Lambda_{\mathrm{f}}=3\right)}=(550 \pm 50) \mathrm{MeV}\right]$ in the framework of the approach in hand.

\section{Conclusion}

In the paper the QCD analytic invariant charge has been applied to study of the quenched lattice simulation data on the static quark-antiquark potential. This strong running coupling enables one to obtain explicitly the confining quarkantiquark potential in the framework of the one-gluon exchange model. A technique for evaluation the integrals of a specific form, developed in this paper, allows one to examine the asymptotic behavior of the derived potential at small and large distances with high accuracy. An explicit formula for the quark-antiquark potential, which interpolates between these asymptotics and satisfies the concavity condition, is obtained. The derived potential has the standard form determined by asymptotic freedom at small distances. At the same time, in the nonperturbative physicallyrelevant region $0.3 \mathrm{fm} \lesssim r \lesssim 1.2 \mathrm{fm}$ this potential agrees fairly well with the lattice simulation data. The value of the parameter $\Lambda_{\mathrm{QCD}}$ is estimated for the case of pure gluodynamics. It is found to be consistent with all the previous estimations of $\Lambda_{\mathrm{QCD}}$ in the framework of the approach developed.

\section{Acknowledgments}

The author is grateful to Professor C. Roiesnel and Professor B. Pire for the fruitful discussions and valuable advises, and to Professor G.S. Bali for supplying the relevant lattice simulation data and useful comments. The author also thanks the

CPHT Ecole Polytechnique for the very warm hospitality. The partial support of RFBR (grants 02-01-00601 and 04-02-81025) and NSh-2339.2003.2 is appreciated.

\section{References}

1. P.J. Redmond, Phys. Rev. 112, 1404 (1958); P.J. Redmond and J.L. Uretsky, Phys. Rev. Lett. 1, 147 (1958).

2. N.N. Bogoliubov, A.A. Logunov, and D.V. Shirkov, Zh. Exp. Teor. Fiz. 37, 805 (1959) [Sov. Phys. JETP 37, 574 (1960)].

3. D.V. Shirkov and I.L. Solovtsov, Phys. Rev. Lett. 79, 1209 (1997).

4. I.L. Solovtsov and D.V. Shirkov, Teor. Mat. Fiz. 120, 482 (1999) [Theor. Math. Phys. 120, 1220 (1999)]; D.V. Shirkov, Eur. Phys. J. C22, 331 (2001).

5. A.V. Nesterenko, Phys. Rev. D62, 094028 (2000).

6. A.V. Nesterenko, Phys. Rev. D64, 116009 (2001).

${ }^{\mathrm{d}}$ It is interesting to note here that a similar values of parameter $\Lambda_{\mathrm{QCD}}$ have also been obtained within different approaches to this issue (see Refs. 19] 27] 32). 
7. A.V. Nesterenko, Int. J. Mod. Phys. A18, 5475 (2003).

8. A.V. Nesterenko, Mod. Phys. Lett. A15, 2401 (2000).

9. A.V. Nesterenko and I.L. Solovtsov, Mod. Phys. Lett. A16, 2517 (2001).

10. G.S. Bali et al., (SESAM and T $\chi \mathrm{L}$ Collaborations), Phys. Rev. D62, 054503 (2000).

11. D.A. Smith and M.J. Teper (UKQCD Collaboration), Phys. Rev. D58, 014505 (1998); A. Ringwald and F. Schrempp, Phys. Lett. B459, 249 (1999); B503, 331 (2001).

12. A.V. Nesterenko, in Proceedings of the Tenth High Energy Physics International Conference in Quantum Chromodynamics, Montpellier, France, 2003 (to be published); arXiv: hep-ph/0307283

13. A.V. Nesterenko, in Proceedings of the Fifth International Conference on Quark Confinement and the Hadron Spectrum, Gargnano, Italy, 2002, edited by N. Brambilla and G. Prosperi (World Scientific, Singapore, 2003), p. 288; arXiv: hep-ph/0210122

14. F. Schrempp, J. Phys. G28, 915 (2002).

15. S. Titard and F.J. Yndurain, Phys. Rev. D49, 6007 (1994).

16. W. Lucha, F.F. Schoberl, and D. Gromes, Phys. Rep. 200, 127 (1991).

17. N. Brambilla and A. Vairo, arXiv: hep-ph/9904330

18. V.V. Kiselev and A.K. Likhoded, Usp. Fiz. Nauk 172, 497 (2002) [Sov. Phys. Usp. 45, $455(2002)]$.

19. R. Levine and Y. Tomozawa, Phys. Rev. D19, 1572 (1979).

20. I.S. Gradshteyn and I.M. Ryzhik, Table of Integrals, Series, and Products, edited by A. Jeffrey (Academic, London, 1994).

21. G. Fogleman, D.B. Lichtenberg, and J.G. Wills, Lett. Nuovo Cimento 26, 369 (1979); D.B. Lichtenberg and J.G. Wills, Nuovo Cimento A47, 483 (1978).

22. M. Melles, Phys. Rev. D62, 074019 (2000).

23. M. Peter, Phys. Rev. Lett. 78, 602 (1997); Nucl. Phys. B501, 471 (1997).

24. V.V. Kiselev, A.E. Kovalsky, and A.I. Onishchenko, Phys. Rev. D64, 054009 (2001); V.V. Kiselev, A.K. Likhoded, O.N. Pakhomova, and V.A. Saleev, ibid. D66, 034030 (2002).

25. E. Seiler, Phys. Rev. D18, 482 (1978); C. Bachas, ibid. D33, 2723 (1986).

26. G.S. Bali, K. Schilling, and A. Wachter, Phys. Rev. D56, 2566 (1997); G.S. Bali and P. Boyle, ibid. D59, 114504 (1999).

27. W. Buchmuller, G. Grunberg, and S.-H. H. Tye, Phys. Rev. Lett. 45, 103 (1980); 45, 587(E) (1980); W. Buchmuller and S.-H. H. Tye, Phys. Rev. D24, 132 (1981).

28. K. Hagiwara, A.D. Martin, and A.W. Peacock, Z. Phys. C33, 135 (1986).

29. R. Akhoury and V.I. Zakharov, Phys. Lett. B438, 165 (1998); G.S. Bali, ibid. B460, 170 (1999)

30. T. Lee, Phys. Rev. D67, 014020 (2003).

31. S.L. Meyer, Data Analysis for Scientists and Engineers (Wiley, New York, 1975).

32. W. Celmaster and F.S. Henyey, Phys. Rev. D18, 1688 (1978). 